\title{
Population pharmacokinetics of pomalidomide in patients with relapsed or refractory multiple myeloma with various degrees of impaired renal function
}

Yan $\mathrm{Li}^{\prime}$

Xiaomin Wang ${ }^{2}$

Edward O’Mara'

Meletios A Dimopoulos ${ }^{3}$

Pieter Sonneveld ${ }^{4}$

Katja C Weisel ${ }^{5}$

Jeffrey Matous ${ }^{6}$

David S Siegel ${ }^{7}$

Jatin J Shah ${ }^{8}$

Elisabeth Kueenburg ${ }^{9}$

Lars Sternas 9

Chloe Cavanaugh ${ }^{9}$

Mohamed Zaki ${ }^{9}$

Maria Palmisano

Simon Zhou'

'Translational Development and Clinical Pharmacology, Celgene Corporation, Summit, NJ, USA; ${ }^{2}$ Non-Clinical

Development and Drug Metabolism and

Pharmacokinetics, Celgene Corporation,

Summit, NJ, USA; ${ }^{3}$ Department of Clinical

Therapeutics, National and Kapodistrian

University of Athens, Athens, Greece;

${ }^{4}$ Department of Hematology, Erasmus

MC Cancer Institute, Rotterdam, the

Netherlands; ${ }^{5}$ Department of Hematology,

University Hospital Tübingen, Tübingen,

Germany; ' ${ }^{6}$ epartment of Hematology/

Oncology, Colorado Blood Cancer

Institute, Denver, CO, USA; ${ }^{7}$ Myeloma

Division, John Theurer Cancer Center,

Hackensack University Medical Center,

Hackensack, NJ, USA; ${ }^{8}$ Department of

Lymphoma/Myeloma, Division of Cancer

Medicine, MD Anderson Cancer Center,

Houston, TX, USA; ' $\mathrm{Global}$ Clinical R\&D,

Celgene Corporation, Summit, NJ, USA

Correspondence: Simon Zhou

Translational Development and Clinical

Pharmacology, Celgene Corporation, 86

Morris Avenue, Summit, NJ 07920, USA

Tel + I 9086739284

Fax + I 9086732842

Email szhou@celgene.com
This article was published in the following Dove Press journal:

Clinical Pharmacology:Advances and Applications

8 November 2017

Number of times this article has been viewed

Abstract: Pomalidomide is an immunomodulatory drug for treatment of relapsed or refractory multiple myeloma (rrMM) in patients who often have comorbid renal conditions. To assess the impact of renal impairment on pomalidomide exposure, a population pharmacokinetics (PPK) model of pomalidomide in rrMM patients with various degrees of impaired renal function was developed. Intensive and sparse pomalidomide concentration data collected from two clinical studies in rMM patients with normal renal function, moderately impaired renal function, severely impaired renal function not requiring dialysis, and with severely impaired renal function requiring dialysis were pooled over the dose range of 2 to $4 \mathrm{mg}$, to assess specifically the influence of the impaired renal function as a categorical variable and a continuous variable on pomalidomide clearance and plasma exposure. In addition, pomalidomide concentration data collected on dialysis days from both the withdrawal (arterial) side and from the returning (venous) side of the dialyzer, from rrMM patients with severely impaired renal function requiring dialysis, were used to assess the extent to which dialysis contributes to the removal of pomalidomide from blood circulation. PPK analyses demonstrated that moderate to severe renal impairment not requiring dialysis has no influence on pomalidomide clearance or plasma exposure, as compared to those patients with normal renal function, while pomalidomide exposure increased approximately $35 \%$ in patients with severe renal impairment requiring dialysis on nondialysis days. In addition, dialysis increased total body pomalidomide clearance from $5 \mathrm{~L} / \mathrm{h}$ to $12 \mathrm{~L} / \mathrm{h}$, indicating that dialysis will significantly remove pomalidomide from the blood circulation. Thus, pomalidomide should be administered post-dialysis on the days of dialysis.

Keywords: hemodialysis, pomalidomide, population pharmacokinetics, renal impairment

\section{Introduction}

The IMiD ${ }^{\circledR}$ agent pomalidomide (Celgene Corporation, Summit, NJ, USA) has pleiotropic cytotoxic effects against multiple myeloma $(\mathrm{MM})$ cells ${ }^{1,2}$ in addition to antiproliferative, ${ }^{3,4}$ antiangiogenic,${ }^{5-7}$ and immunomodulatory activity. ${ }^{8,9}$ Furthermore, pomalidomide has potent effects on key cytokines, including IL-10, IFN $\gamma$, and TNF $\alpha .^{10}$ Pomalidomide has been studied for treatment of various hematologic and non-neoplastic hematologic disorders ${ }^{11-13}$ and is approved (in combination with dexamethasone) in the European Union and United States for the treatment of patients with MM who have received $\geq 2$ prior therapies, including lenalidomide and bortezomib (in the European Union; a proteasome inhibitor in the United States), and who have progressed on or within 60 days of completion of the last therapy or have disease progression on the submit your manuscript Dovepress if in $>$ 
last therapy. ${ }^{14,15}$ This combination (pomalidomide plus lowdose dexamethasone) significantly increased progression-free survival and overall survival compared with high-dose dexamethasone. ${ }^{13}$ Thrombocytopenia, neutropenia, and anemia were the most common grade $3 / 4$ adverse events.

Lenalidomide, pomalidomide's IMiD analog, is metabolized minimally and eliminated predominantly via urinary excretion in the unchanged form in humans,${ }^{16}$ resulting in dosage adjustments for patients with creatinine clearance $(\mathrm{CrCl})<50 \mathrm{~mL} / \mathrm{min}^{17,18}$ Pomalidomide is eliminated predominantly through renal excretion ( $73 \%$ of the administered dose), with $<3 \%$ of the administered dose excreted in urine as unchanged pomalidomide across all dose levels. ${ }^{19}$ Thus, it is relevant to assess the influence of renal impairment on pomalidomide clearance and plasma exposure.

Pomalidomide population pharmacokinetics (PPK) was previously assessed in both healthy individuals and patients with relapsed or refractory MM (rrMM).$^{20}$ Those analyses suggested that pomalidomide systemic clearance was comparable between the two groups. Covariate analysis showed pomalidomide clearance was unaffected by demographic factors, with the exception of sex, which had a difference $<30 \%$, making its clinical relevance unlikely. Furthermore, pomalidomide clearance was not significantly affected by renal function/impairment (as measured by $\mathrm{CrCl}$ between $20-188 \mathrm{~mL} / \mathrm{min})$.

In a previous PPK analysis, the data set included only a few patients with moderately or severely impaired renal function. Thus, there is a need for a thorough assessment of the influence of impaired renal function on pomalidomide clearance, including more data from patients with moderately or severely impaired renal function.

Following the PPK analysis, two studies were conducted to assess the specific effect of renal function on pharmacokinetics (PK), safety, and/or efficacy of pomalidomide in patients with renal impairment. CC-4047-MM-008 is an ongoing Phase 1, multicenter, open-label, dose-escalation study. ${ }^{21}$ The primary objective of the study was to determine the $\mathrm{PK}$ and safety of pomalidomide when given in combination with low-dose dexamethasone in patients with rMM and impaired renal function. CC-4047-MM-013 is an ongoing Phase 2, multicenter, open-label study to determine the efficacy and safety of pomalidomide in combination with low-dose dexamethasone in patients with rrMM and moderate or severe renal impairment, including patients undergoing hemodialysis. ${ }^{22}$ Secondary objectives included evaluating the PK of pomalidomide in patients with various degrees of renal impairment.

The aim of this study was to use the cumulative data of patients with rrMM and degrees of renal impairment comorbidity from CC-4047MM-008 and CC-4047-MM-013 to evaluate the relationship of renal function measures (treated as both a categorical variable and a continuous variable) with pomalidomide clearance to assess the extent to which hemodialysis contributes to the elimination of pomalidomide and to optimize the start time of hemodialysis after pomalidomide administration by simulation.

\section{Methods}

\section{Study population and PK sampling}

The PPK analysis utilized data from 63 participants in two clinical studies, CC-4047-MM-008 and CC-4047-MM-013. In the PPK data set, there is an adequate distribution of patients with rMM and various degrees of impaired renal function (Table 1). In both studies, once-daily pomalidomide was administered orally. Institutional review boards or ethics committees (Table S1) approved both studies, and all study participants provided written informed consent.

\section{Bioanalytical methodology}

To determine human plasma samples for pomalidomide concentrations, a validated liquid chromatography-tandem mass

Table I Number of patients with various degrees of impaired renal function included in the population pharmacokinetic analysis

\begin{tabular}{|c|c|c|c|c|}
\hline \multirow[t]{2}{*}{ Renal function group } & \multirow[t]{2}{*}{ Classification of renal function } & \multicolumn{3}{|c|}{ Number of patients } \\
\hline & & CC-4047-MM-008 & CC-4047-MM-0I3 & Total \\
\hline Patients with normal renal function & $\mathrm{CrCl} \geq 60 \mathrm{~mL} / \mathrm{min}$ & 8 & - & 8 \\
\hline $\begin{array}{l}\text { Patients with moderately impaired renal } \\
\text { function }\end{array}$ & $30<\mathrm{eGFR}<45 \mathrm{~mL} / \mathrm{min} / 1.73 \mathrm{~m}^{2}$ & - & 15 & 15 \\
\hline $\begin{array}{l}\text { Patients with severely impaired renal } \\
\text { function not requiring hemodialysis }\end{array}$ & $\begin{array}{l}\mathrm{CrCl}<30 \mathrm{~mL} / \mathrm{min} \text { or eGFR }<30 \mathrm{~mL} / \\
\mathrm{min} / 1.73 \mathrm{~m}^{2} \text { not requiring dialysis }\end{array}$ & 11 & 19 & 30 \\
\hline $\begin{array}{l}\text { Patients with severely impaired renal } \\
\text { function requiring hemodialysis }\end{array}$ & $\begin{array}{l}\mathrm{CrCl}<30 \mathrm{~mL} / \mathrm{min} \text { or eGFR }<30 \mathrm{~mL} / \\
\mathrm{min} / \mathrm{l} .73 \mathrm{~m}^{2} \text { requiring dialysis }\end{array}$ & I & 9 & 10 \\
\hline Total & & 20 & 43 & 63 \\
\hline
\end{tabular}

Abbreviations: $\mathrm{CrCl}$, creatinine clearance; eGFR, estimated glomerular filtration rate. 
spectrometry (LC-MS/MS) assay was used. The lower limit of quantification was $0.25 \mathrm{ng} / \mathrm{mL}$. As an internal standard, plasma samples were spiked with stable labeled pomalidomide. These were subsequently processed by liquid-liquid extraction and then analyzed using reversed-phase highperformance liquid chromatography (HPLC) with electrospray MS/MS detection. HPLC was used to separate peaks.

\section{Population PK model building}

A nonlinear mixed-effect modeling program (NONMEM version 7.2; Icon Development Solutions, Ellicott City, MD, USA) was used for pomalidomide concentration-time PK analysis. Graphic processing was performed using S-Plus (version 8.2; TIBCO Software Inc, Somerville, MA, USA) and R-based model-building aid Perl-Speaks-NONMEM (PsN, version 3.5.3; Kajsa Harling and Andrew Hooker, Uppsala University, Uppsala, Sweden) post-processing software. Structural model comparisons were based on goodnessof-fit criteria and objective function value.

Selection of the most appropriate model was based on prior knowledge of pomalidomide disposition, graphical analysis, and statistical evaluation. Pomalidomide concentrationtime data, per visual inspection and data-fitting criteria, were best described by a one-compartment structure PK model. This model featured the first-order absorption rate constant (Ka), absorption lag time, apparent central compartment volume of distribution, and apparent clearance (CL/F).

The inter-individual variability (IIV) in PK parameters assumed a log-normal distribution and was modeled as follows:

$$
P_{i}=P \cdot e^{\eta i}
$$

with $P_{i}$ as the parameter value for the $i$ th individual, $P$ as the typical parameter value in the population, and $\eta_{i}$ as a random inter-individual parameter effect for the $i$ th participant with a mean $=0$ and variance $\omega^{2}$ (ie, $\eta \sim N\left[0, w^{2}\right]$ ).

IIV or residual variability was modeled as follows:

$$
\ln \left(C_{i j}\right)=\ln \left(C_{m i j}\right)+\varepsilon_{i j}
$$

with $C_{i j}$ and $C_{m i j}$ as the observed and model-predicted $j$ th concentration in the $i$ th participant, respectively, and $\varepsilon_{i j}$ as the random residual effect for the $j$ th concentration in the $i$ th participant with a mean $=0$ and variance of $\sigma^{2}$.

Visual predictive check (VPC) was used for model evaluation. This compared model predictions with observations and provided an evaluation of model assumption and population parameter estimates. The final PPK model's ability to describe observed concentration data was tested using simulations; VPCs were performed using 1000 data sets using the same dosages, dosing schedules, and sampling times as in the original data set.

The nonparametric bootstrap approach was used to evaluate the stability of the final PK parameter estimates and 90\% CI. There were 500 bootstrap data sets, and the final model was fit to each. Every model parameter was estimated for each set; $96 \%$ of the bootstrap runs were successful. For each of the 500 estimates, median and nonparametric $90 \%$ CI (5th-95th percentiles) were calculated for each parameter.

\section{Covariate analysis}

Renal function was the key covariate for the covariate analysis. Both the Cockcroft-Gault equation ${ }^{23}$ and the Modification of Diet in Renal Disease (MDRD) equation ${ }^{24,25}$ have been used to calculate $\mathrm{CrCl}$ and eGFR:

$\mathrm{CrCl}=[(140-$ age $) \times$ body weight $] /(72 \times$ serum creatinine level $) \times(0.85$ for females $)$

(Equation 3)

$\mathrm{CrCl}$ is capped at a physiological level of $150 \mathrm{~mL} / \mathrm{min}$.

eGFR $\left(\mathrm{mL} / \mathrm{min} / 1.73 \mathrm{~m}^{2}\right)=175 \times($ Scr,std $)-1.154 \times($ age $)$ $-0.203 \times(0.724$ if female $) \times(1.212$ if African American $)$

(Equation 4)

with Scr,std as serum creatinine level measured with a standardized assay.

The influence of renal function (treated as both a categorical variable and a continuous variable) on pomalidomide clearance was specifically assessed in the covariate analysis. Other typical covariates in the PPK data set included, but were not limited to, age, weight, body surface area, sex, and hepatic function markers.

The pomalidomide covariate model was developed using the stepwise covariate model-building tool of PsN. The stepwise covariate model has a fixed set of PK parametercovariate relations defined within it; predefined shapes for the pomalidomide continuous covariate model development parameter-covariate relations included either linear or power equations.

In the pomalidomide covariate model development, categorical covariates were included as follows:

$$
P=\theta \cdot\left(1+\theta_{\text {cov }} \cdot Z_{i n d, k}\right)
$$

(Equation 5)

with $\theta_{\text {cov }}$ representing the coefficient for the covariate effect and $Z_{i n d, k}$ as an indicator variable representing one binary covariate form. 


\section{Monte Carlo simulation}

Based on the developed PPK models in which renal function was treated as both a categorical variable and a continuous variable, Monte Carlo simulations were performed using NONMEM 7.2 to investigate whether impaired renal function would alter the plasma pomalidomide exposures. The plasma concentration profiles for patients with rrMM with various degrees of impaired renal function were simulated at steady state, and then the drug exposures were calculated by non-compartmental analysis for comparison.

\section{Assessing effect of hemodialysis on pomalidomide PK}

Hemodialysis may affect pomalidomide PK to an extent that necessitates dosage adjustment. The need for dosage adjustment arises when a significant fraction of pomalidomide is removed by hemodialysis. In such cases, a change in the dosage regimen, such as a supplemental dose following hemodialysis, may be appropriate.

The hemodialysis clearance $\left(\mathrm{CL}_{\mathrm{D}}\right)$ was estimated from the plasma concentration-time data collected on hemodialysis days from both the withdrawal ("arterial") and returning ("venous") sides of the dialyzer in patients requiring hemodialysis:

$$
\mathrm{CL}_{\mathrm{D}}=\mathrm{Q} *\left(\mathrm{C}_{\mathrm{a}}-\mathrm{C}_{\mathrm{v}}\right) / \mathrm{C}_{\mathrm{a}} \quad \text { (Equation 6) }
$$

with $\mathrm{C}_{\mathrm{a}}$ as concentration in plasma entering the dialyzer (arterial line), $\mathrm{C}_{\mathrm{v}}$ as concentration in plasma exiting the dialyzer (venous line), and Q as the flow rate of blood through the dialyzer.

$\mathrm{CL}_{\mathrm{D}}$ was compared with pomalidomide plasma clearance to assess the effect of hemodialysis on pomalidomide PK. Simulations were conducted to identify dosages and the starting time of hemodialysis after pomalidomide administration that achieved the goal for patients requiring hemodialysis.

\section{Results}

\section{Analysis populations and demographics}

Sixty-three participants with various degrees of impaired renal function were included in the final PPK analysis data set (Table 1). Baseline characteristics are summarized in Table 2. The participants had a median age of 69 (range, 46-86) years. The median $\mathrm{CrCl}$ and the median eGFR were 28.3 (range, 8.7-115.4) $\mathrm{mL} / \mathrm{min}$ and 27 (range, $5-84$ ) $\mathrm{mL} / \mathrm{min} / 1.73 \mathrm{~m}^{2}$, respectively.
Table 2 Demographic characteristics of analysis population

\begin{tabular}{|c|c|c|}
\hline Variable & Study & $\begin{array}{l}\text { Value, median } \\
\text { (min, max) }\end{array}$ \\
\hline \multirow[t]{3}{*}{ Age (years) } & CC-4047-MM-0I3 & $71(55,86)$ \\
\hline & CC-4047-MM-008 & $67(46,84)$ \\
\hline & Total & $69(46,86)$ \\
\hline \multirow[t]{3}{*}{ ALT (U/L) } & CC-4047-MM-0I3 & $15(8,58)$ \\
\hline & CC-4047-MM-008 & $21(6,47)$ \\
\hline & Total & $17(6,58)$ \\
\hline \multirow[t]{3}{*}{ ALB $(g / L)$} & CC-4047-MM-0I3 & $36.4(0,45.6)$ \\
\hline & CC-4047-MM-008 & $37.5(30,42)$ \\
\hline & Total & $37(0,45.6)$ \\
\hline \multirow[t]{3}{*}{ ALP (U/L) } & CC-4047-MM-0I3 & $78(38,1154)$ \\
\hline & CC-4047-MM-008 & $70.5(39,177)$ \\
\hline & Total & $76(38,1154)$ \\
\hline \multirow[t]{3}{*}{ AST (U/L) } & CC-4047-MM-0I3 & $21(10,68)$ \\
\hline & CC-4047-MM-008 & $26.5(13,43)$ \\
\hline & Total & $22(10,68)$ \\
\hline \multirow[t]{3}{*}{ Bilirubin $(\mu \mathrm{mol} / \mathrm{L})$} & CC-4047-MM-0I3 & $6.8(3,18)$ \\
\hline & CC-4047-MM-008 & $6.8(5.1,12)$ \\
\hline & Total & $6.8(3,18)$ \\
\hline \multirow[t]{3}{*}{ Body mass index } & CC-4047-MM-0I3 & $26.6(19.6,38)$ \\
\hline & CC-4047-MM-008 & $28.3(19.6,36.9)$ \\
\hline & Total & $26.8(19.6,38)$ \\
\hline \multirow[t]{3}{*}{ Body weight (kg) } & CC-4047-MM-0I3 & $74.4(4 \mid .2, I 10)$ \\
\hline & CC-4047-MM-008 & $83.9(39.9,116.6)$ \\
\hline & Total & $78.2(39.9,116.6)$ \\
\hline \multirow[t]{3}{*}{$\mathrm{CrCl}(\mathrm{mL} / \mathrm{min})$} & CC-4047-MM-0I3 & $26.1(8.7,73.7)$ \\
\hline & CC-4047-MM-008 & $32.3(12.3,115.4)$ \\
\hline & Total & $28.3(8.7,115.4)$ \\
\hline eGFR (mL/min/ & CC-4047-MM-0I3 & $25.8(5,45 . I)$ \\
\hline \multirow[t]{2}{*}{$\left.1.73 \mathrm{~m}^{2}\right)$} & CC-4047-MM-008 & $29(5,84)$ \\
\hline & Total & $27(5,84)$ \\
\hline \multirow[t]{3}{*}{ Height (cm) } & CC-4047-MM-0I3 & $168(132,194)$ \\
\hline & CC-4047-MM-008 & 169.I (136.9, I89.8) \\
\hline & Total & $168(132,194)$ \\
\hline \multirow[t]{3}{*}{ LDH (U/L) } & CC-4047-MM-0I3 & $227(115,1684)$ \\
\hline & CC-4047-MM-008 & $391.5(88,1954)$ \\
\hline & Total & $262(88,1954)$ \\
\hline \multirow[t]{3}{*}{ Total protein $(\mathrm{g} / \mathrm{L})$} & CC-4047-MM-0I3 & $63(0.1,113)$ \\
\hline & CC-4047-MM-008 & $73.5(52,107)$ \\
\hline & Total & $68(0.1,113)$ \\
\hline
\end{tabular}

Abbreviations: ALB, albumin; ALT, alanine aminotransferase; $A L P$, alkaline phosphatase; AST, aspartate aminotransferase; $\mathrm{CrCL}$, creatinine clearance; eGFR, estimated glomerular filtration rate; LDH, lactate dehydrogenase.

\section{Assessment of the relationship of measures of renal function (treated as categorical variable) and the pomalidomide clearance}

Visual examination of the dose-normalized average concentration vs time profiles by renal function group (Figure 1) showed similar pomalidomide concentrations among patients 
with $\mathrm{rrMM}$ with normal renal function $(\mathrm{CrCl} \geq 60 \mathrm{~mL} /$ $\mathrm{min})$, with moderate renal impairment $(30<\mathrm{eGFR} \leq 45 \mathrm{~mL} /$ $\min / 1.73 \mathrm{~m}^{2}$ ), with severe renal impairment not requiring hemodialysis $(\mathrm{CrCl}<30 \mathrm{~mL} / \mathrm{min}$ or eGFR $<30 \mathrm{~mL} /$ $\min / 1.73 \mathrm{~m}^{2}$ ), and with severe renal impairment requiring hemodialysis.

Pomalidomide concentration-time profiles were adequately described by a one-compartment model with firstorder absorption. Renal function was incorporated as a categorical variable to assess the relationship of renal function measures and pomalidomide clearance. None of the other tested covariates were significant enough to be included in the final model using the stepwise covariate model based on the predefined statistical criteria for inclusion of a covariate.
The PPK parameters from the final PPK model are presented in Table 3 . The model parameters were precisely estimated with the base model as observed from the narrow $90 \%$ bootstrap CI. The estimated IIV and associated covariance were reasonably precise.

The bootstrap replicates showed similar median values of the parameters with narrow $90 \%$ CIs, compared with the original NONMEM estimates (Table 3 ). These results suggest that the final model is stable and robust.

As shown in Figure 2, VPC evaluation demonstrated that the $90 \%$ prediction intervals of the median, 5 th, and 95th percentiles of simulated results could cover the observed data percentiles appropriately, and no obvious bias was noted. Overall, the observed pomalidomide

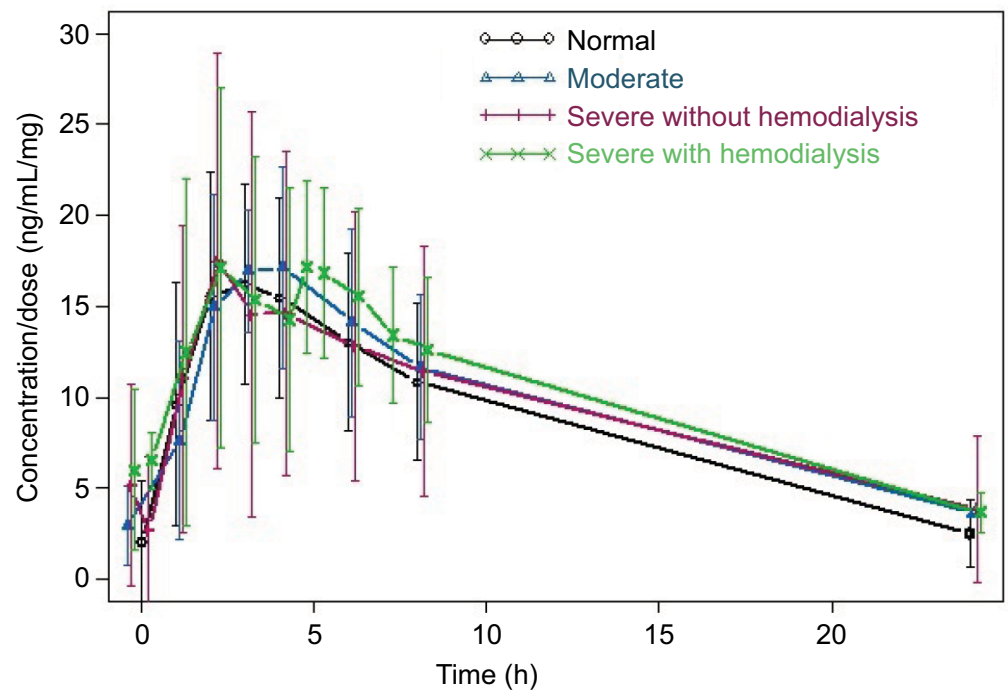

Figure I Average dose-normalized pomalidomide concentration vs time profiles by renal function group.

Table 3 Pharmacokinetic parameter estimates from the final model with renal function treated as a categorical variable

\begin{tabular}{llll}
\hline PK parameter & Estimate & Bootstrap median estimate & $\mathbf{9 0 \%}$ bootstrap Cla \\
\hline $\mathrm{Ka}(\mathrm{I} / \mathrm{h})$ & 0.724 & 0.774 & $0.533-1.23$ \\
$\mathrm{~V} / \mathrm{F}(\mathrm{L})$ & 58.300 & 58.521 & $51.597-66.055$ \\
$\mathrm{CL} / \mathrm{F}$ normal $(\mathrm{L} / \mathrm{h})$ & 5.130 & 5.159 & $4.283-6.069$ \\
$\mathrm{CL} / \mathrm{F}$ ratio (group 2 vs group I) & 1.020 & 1.019 & $0.848-1.195$ \\
$\mathrm{CL} / \mathrm{F}$ ratio (group 3 vs group I) & 1.010 & 1.018 & $0.87 \mathrm{I}-1.169$ \\
$\mathrm{CL} / \mathrm{F}$ ratio (group 4 vs group I) & 0.724 & 0.729 & $0.606-0.844$ \\
Tlag (h) & 0.154 & 0.183 & $0.002-0.43$ \\
$\omega^{2}(\mathrm{Ka})$ & 0.782 & 0.804 & $0.44 I-1.387$ \\
$\omega^{2}(\mathrm{~V} / \mathrm{F})$ & 0.139 & 0.136 & $0.069-0.236$ \\
$\omega(\mathrm{V} / \mathrm{F}): \omega(\mathrm{CL} / \mathrm{F})$ & 0.163 & 0.156 & $0.088-0.236$ \\
$\omega^{2}(\mathrm{CL} / \mathrm{F})$ & 0.198 & 0.189 & $0.121-0.273$ \\
$\delta^{2}$ & 0.341 & 0.321 & $0.194-0.459$ \\
\hline
\end{tabular}

Notes: $\mathrm{CL} / \mathrm{F}_{\text {normal }}$, apparent clearance for patients with normal renal function; Ka, absorption rate constant; Tlag, lag time; V/F, apparent volume of distribution; $\omega^{2}$, variance of interpatient variability; $\delta^{2}$, variance of intra-patient variability. Group I represents patients with normal renal function; group 2 represents patients with moderately impaired renal function; group 3 represents patients with severely impaired renal function not requiring hemodialysis; and group 4 represents patients with severely impaired renal function requiring hemodialysis. ${ }^{\text {aBootstrap }} \mathrm{Cl}$ values were taken from bootstrap calculation.

Abbreviation: PK, pharmacokinetics. 

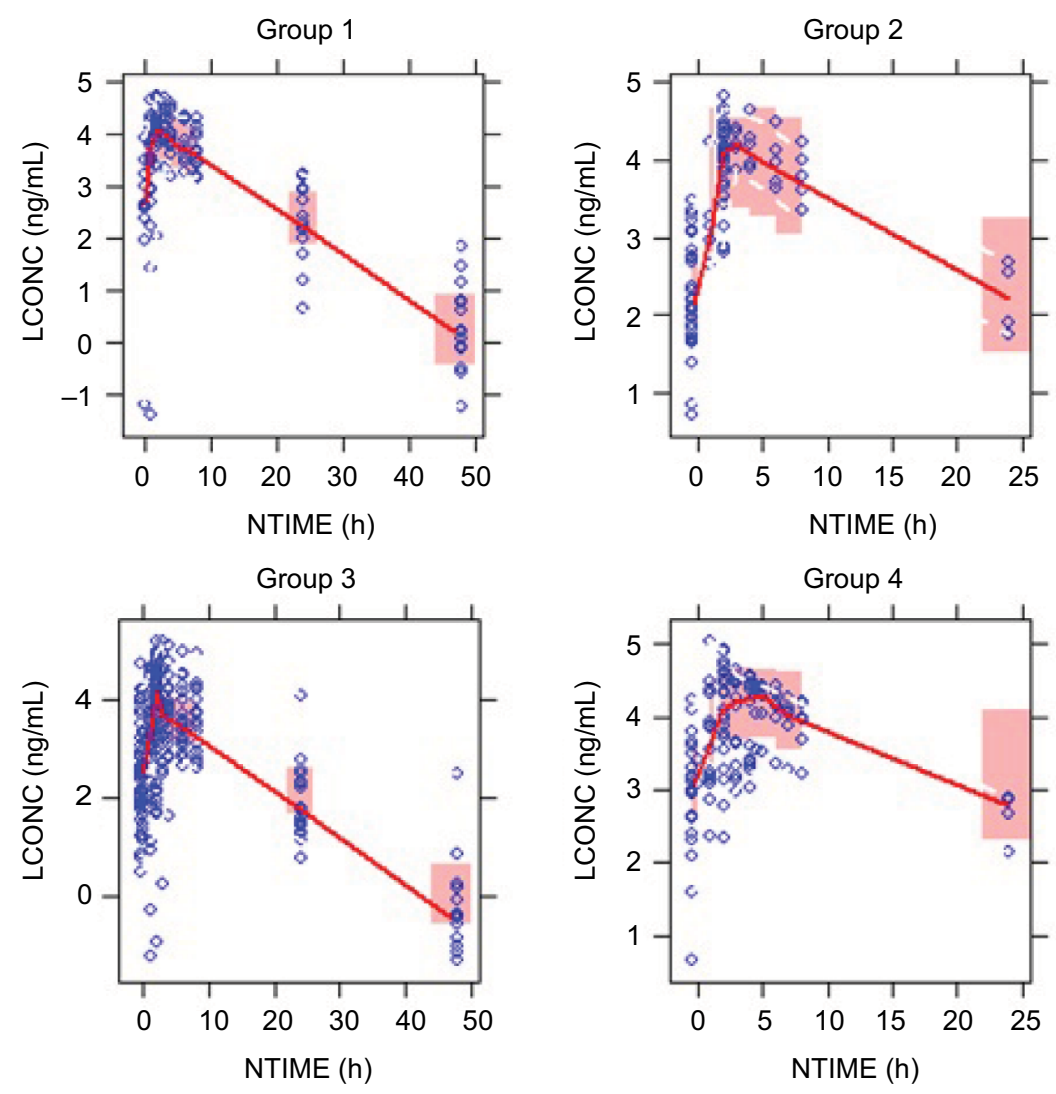

Figure 2 Visual predictive checks for time profiles of pomalidomide concentrations by group.

Notes: Group I: patients with normal renal function; Group 2: patients with moderately impaired renal function; Group 3: patients with severely impaired renal function not requiring hemodialysis; Group 4: patients with severely impaired renal function requiring hemodialysis. Circles represent observed data. Lines represent the 50th (solid) percentiles of the observed data. Shaded areas represent nonparametric $95 \% \mathrm{Cl}$ about the 50th (pink) percentiles for the corresponding model-predicted percentiles.

Abbeviations: LCONC, logarithm-transformed concentration; NTIME, nominal time.

concentration variability was adequately described by the estimated IIV.

Taken together, the final PPK model adequately characterized pomalidomide concentrations in the log range of -1.3666 to 5.196 (ie, $0.255-180.569 \mathrm{ng} / \mathrm{mL}$ ). This enabled the use of the model to predict pomalidomide exposure for Monte Carlo simulations, making the final PPK model appropriate.

\section{Developing dosage recommendation for patients with rrMM with different levels of renal function by Monte Carlo}

\section{simulation}

Per regulatory agencies' guidance, specific dosing recommendations should be constructed based on the study results using the aforementioned model for the relationships between renal function and relevant PK parameters. Simulations are encouraged as a means to identify dosages and dosing intervals for patients with different levels of renal function.
Monte Carlo simulations were conducted based on the final PPK model in which renal function was treated as a four-level categorical variable. Fifty patients with various degrees of renal impairment (normal renal function, moderately impaired renal function, severely impaired renal function not requiring hemodialysis, and severely impaired renal function requiring hemodialysis) were included in each virtual trial. Two hundred virtual trials were performed using Monte Carlo simulations.

The mean values of the plasma concentration profiles at steady state obtained from 200 Monte Carlo simulations were not remarkably different among patients with various degrees of renal impairment (Figure 3 ). The mean values of the plasma exposures (area under the plasma concentrationtime curve) obtained from the simulations were not remarkably different among patients with normal renal function, moderately impaired renal function, and severely impaired renal function not requiring hemodialysis. Patients with severely impaired renal function requiring hemodialysis 
showed $35.8 \%$ higher exposure compared with patients with normal renal function (Table 4).

\section{Assessment of the relationship of renal function measures of $\mathrm{CrCl}$ or eGFR and pomalidomide clearance}

Historically, the Cockcroft-Gault equation for calculating $\mathrm{CrCl}$ has been widely used to define the degree of renal impairment, and it is used commonly in the application of drug dosing guidance for patients with renal impairment. In the CC-4047-MM-008 study, $\mathrm{CrCl}$ was used to define the degree of renal impairment. Recently, the MDRD eGFR equation has increasingly been used as the standard in clinical practice to assess renal function. In the CC-4047-MM-013 study, eGFR was used to define the degree of renal impairment. Therefore, it is critical to assess the relationship between renal function treated as a continuous variable $(\mathrm{CrCl}$ or eGFR) and pomalidomide clearance.

As shown in Figure 4, pomalidomide clearance increased with increasing $\mathrm{CrCl}$ or eGFR and becomes constant when the $\mathrm{CrCl}$ is larger than approximately $40 \mathrm{~mL} / \mathrm{min}$ or when the eGFR is larger than approximately $25 \mathrm{~mL} / \mathrm{min} / 1.73 \mathrm{~m}^{2}$. The

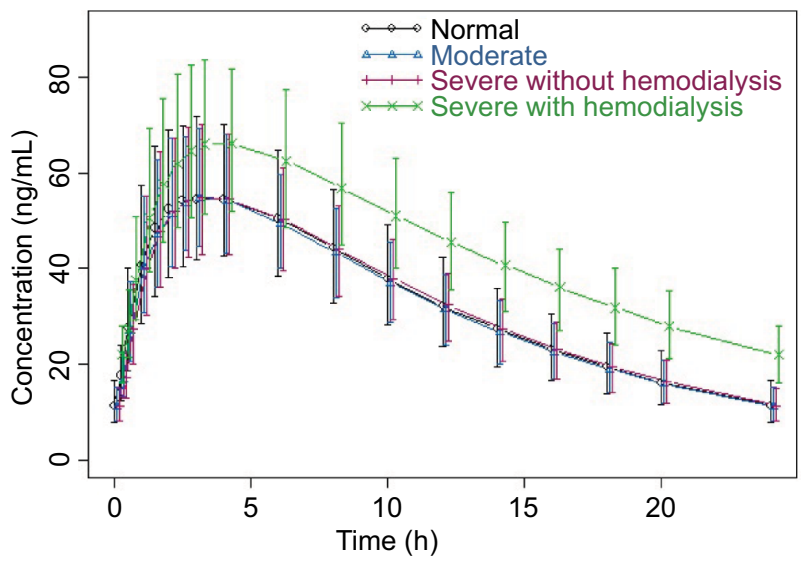

Figure 3 Median values of the plasma concentration profiles with $90 \% \mathrm{Cl}$ from 200 Monte Carlo simulations based on the final population pharmacokinetic model with renal function treated as a categorical variable. reverse-hockey-stick equation was selected to characterize the relationship between pomalidomide clearance and $\mathrm{CrCl}$ or eGFR:

\section{$\mathrm{CL} / \mathrm{F}=$ intercept + slope $\times \mathrm{CrCl}$ or eGFR (when $\mathrm{CrCl}$ or $\mathrm{eGFR} \leq \mathrm{CrCl}_{0}$ or $\mathrm{eGFR}_{0}$ ) $=$ constant $\left(\right.$ when $\mathrm{CrCl}$ or eGFR $>\mathrm{CrCl}_{0}$ or eGFR $)$}

(Equation 7)

Based on goodness-of-fit and statistical criteria, a onecompartment model with $\mathrm{Ka}$ and a reverse-hockey-stick relationship between $\mathrm{CL} / \mathrm{F}$ and $\mathrm{CrCl}$ or eGFR adequately described pomalidomide PK (data not shown) and was chosen for the final structural PPK model (Table 5).

\section{Assessment of the extent to which hemodialysis contributes to the elimination of pomalidomide}

Because pomalidomide is loosely bound to plasma proteins $(12 \%-44 \%)$, the effect of hemodialysis on pomalidomide PK was assessed in patients with end-stage renal disease undergoing hemodialysis on both hemodialysis and nondialysis days to determine the extent to which hemodialysis contributed to the elimination of pomalidomide in both the CC-4047-MM-008 and CC 4047-MM-013 studies. $\mathrm{CL}_{\mathrm{D}}$ was calculated and the median value from seven patients was approximately $12 \mathrm{~L} / \mathrm{h}$, which is higher than pomalidomide total body clearance $(5 \mathrm{~L} / \mathrm{h})$, indicating that the hemodialysis procedure removed pomalidomide from circulation. Therefore, it is critical to optimize the starting time of hemodialysis relative to pomalidomide administration. Simulations based on the final PPK model and observed $\mathrm{CL}_{\mathrm{D}}$ were conducted under two different scenarios.

- Scenario 1: hemodialysis begins after pomalidomide administration.

- Scenario 2: hemodialysis is completed before pomalidomide administration.

Table 4 Summary of systemic pomalidomide exposure (AUC) for patients with various degrees of renal impairment from 200 Monte Carlo simulations based on the final population pharmacokinetic model with renal function treated as a categorical variable

\begin{tabular}{llllll}
\hline Group & Classification of renal function & $\begin{array}{l}\text { Mean of AUC } \\
(\mathbf{n g} / \mathbf{m L} \cdot \mathbf{h})\end{array}$ & $\begin{array}{l}\mathbf{9 0 \%} \mathbf{C l} \text { of AUC } \\
(\mathbf{n g} / \mathbf{m L} \cdot \mathbf{h})\end{array}$ & $\begin{array}{l}\text { Mean of normalized } \\
\text { AUC (\%) }\end{array}$ & $\begin{array}{l}\mathbf{9 0 \%} \mathbf{C l} \text { of normalized } \\
\text { AUC (\%) }\end{array}$ \\
\hline Group 1 & $60<\mathrm{CrCl}<120 \mathrm{~mL} / \mathrm{min}$ & 787.8 & $601.9-1025.9$ & 100.0 & $76.4-130.2$ \\
Group 2 & $30<\mathrm{CrCl}<60 \mathrm{~mL} / \mathrm{min}$ & 773.5 & $609.8-949.9$ & 98.2 & $77.4-120.6$ \\
Group 3 & $15<\mathrm{CrCl}<30 \mathrm{~mL} / \mathrm{min}$ & 789.7 & $628.3-1000.7$ & 100.2 & $79.7-127.0$ \\
Group 4 & $0<\mathrm{CrCl}<15 \mathrm{~mL} / \mathrm{min}$ & 1070.0 & $846.9-1339.5$ & 135.8 & $107.5-170.0$ \\
\hline
\end{tabular}

Notes: Group I represents patients with normal renal function; group 2 represents patients with moderately impaired renal function; group 3 represents patients with severely impaired renal function not requiring hemodialysis; and group 4 represents patients with severely impaired renal function requiring hemodialysis. Abbreviations: AUC, area under the plasma concentration-time curve from 0 to $24 \mathrm{~h} ; \mathrm{CrCl}$, creatinine clearance. 

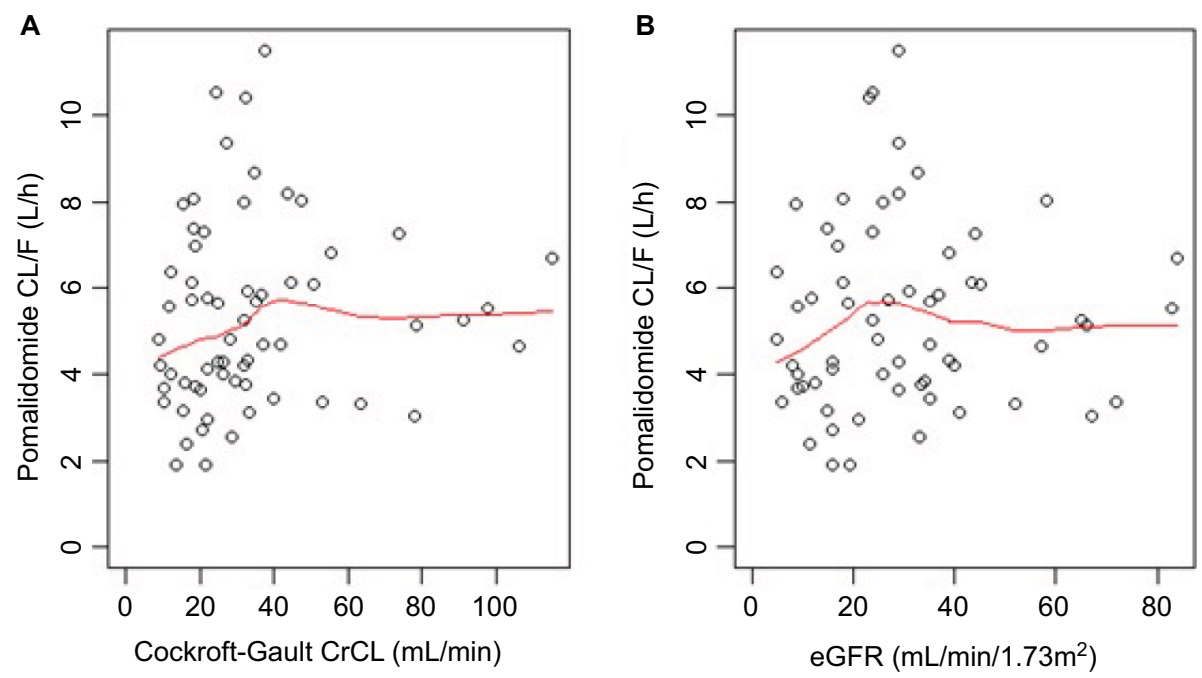

Figure 4 Relationship between pomalidomide apparent clearance (CL/F) and $\mathrm{CrCl}(\mathbf{A})$ or eGFR (B).

Note: Red lines represent the locally weighted scatterplot smoothing line.

Abbreviations: $\mathrm{CrCl}$, creatinine clearance; eGFR, estimated glomerular filtration rate.

Table 5 Pharmacokinetic parameter estimates from the final model with renal function treated as a continuous variable

\begin{tabular}{|c|c|c|c|c|}
\hline \multirow[t]{2}{*}{ PK parameter } & \multicolumn{2}{|c|}{$\mathrm{CrCl}$ as renal function marker } & \multicolumn{2}{|c|}{ eGFR as renal function marker } \\
\hline & Estimate & $95 \%$ bootstrap $\mathrm{Cl}^{\mathrm{a}}$ & Estimate & $90 \%$ bootstrap $\mathrm{Cl}^{\mathrm{a}}$ \\
\hline $\mathrm{Ka}(\mathrm{I} / \mathrm{h})$ & 0.68 & $0.522-0.931$ & 0.678 & $0.526-0.948$ \\
\hline $\mathrm{V} / \mathrm{F}(\mathrm{L})$ & 60.7 & $54.393-68.717$ & 60.5 & $54.502-69.225$ \\
\hline Intercept (L/h) & 3.71 & $2.321-4.796$ & 3.96 & $2.577-5.086$ \\
\hline Slope & 0.0469 & $0.013-0.095$ & 0.0483 & $0.001-0.115$ \\
\hline $\mathrm{CrCl}_{0}(\mathrm{~mL} / \mathrm{min})$ or eGFR $\left(\mathrm{mL} / \mathrm{min} / 1.73 \mathrm{~m}^{2}\right)$ & 37.7 & $35.027-50.343$ & 26.0 & $25.01-30.899$ \\
\hline$\omega^{2}(\mathrm{Ka})$ & 0.986 & $0.638-1.467$ & 0.99 & $0.64-1.46$ \\
\hline$\omega^{2}(\mathrm{~V} / \mathrm{F})$ & 0.0293 & $0-0.081$ & 0.0278 & $0-0.082$ \\
\hline$\omega^{2}$ (intercept) & 0.163 & $0.076-0.383$ & 0.147 & $0.062-0.297$ \\
\hline$\omega^{2}$ (slope) & 0.3 & $0-0.813$ & 0.516 & $0-3.837$ \\
\hline$\delta^{2}$ & 0.351 & $0.2|7-0.47|$ & 0.351 & $0.221-0.492$ \\
\hline
\end{tabular}

Notes: Ka, absorption rate constant; V/F, apparent volume of distribution; $\omega^{2}$, variance of inter-patient variability; $\delta^{2}$, variance of intra-patient variability. ${ }^{\text {BB }}$ ootstrap Cl values were taken from bootstrap calculation.

Abbreviations: $\mathrm{CrCl}$, creatinine clearance; eGFR, estimated glomerular filtration rate; PK, pharmacokinetics.

The simulated concentration vs time profiles under $4 \mathrm{~h}$ hemodialysis for these two scenarios are presented in Figure 5 and Figure 6, respectively. In scenario 1, pomalidomide exposures on hemodialysis days were significantly lower than on nondialysis days under different starting times of hemodialysis. Quantitative assessment suggested that the pomalidomide exposures for scenario 1 were approximately $50 \%-70 \%$ of those on nondialysis days and were likely to result in insufficient pomalidomide exposures on hemodialysis days. On the other hand, in scenario 2, pomalidomide exposure was approximately $83 \%-91 \%$ of that on nondialysis days and provided adequate pomalidomide exposures for patients with severely impaired renal function requiring hemodialysis. When a $6 \mathrm{~h}$ hemodialysis procedure simulation was conducted, similar results were obtained (data not shown).

\section{Discussion}

A quantitative PPK analysis of pomalidomide PPK in both healthy individuals and patients with rrMM has been previously reported. ${ }^{20}$ That study, which investigated the associated IIV and residual variability and assessed the effect of covariates of interest, reported that both groups showed comparable plasma exposures. However, patients with rrMM showed longer duration of terminal-phase plasma concentrations, indicating a deeper pomalidomide tissue/organ distribution. Previously, it was shown within the graphic analysis that, although renal function (determined by $\mathrm{CrCl}$ ) appeared to have a positive correlation with $\mathrm{CL} / \mathrm{F}$, the predefined statistical significance level was not reached in the univariate covariate analysis. In patients with normal, mild, and moderate renal impairment, the geometric mean $\mathrm{CL} / \mathrm{F}$ appeared 


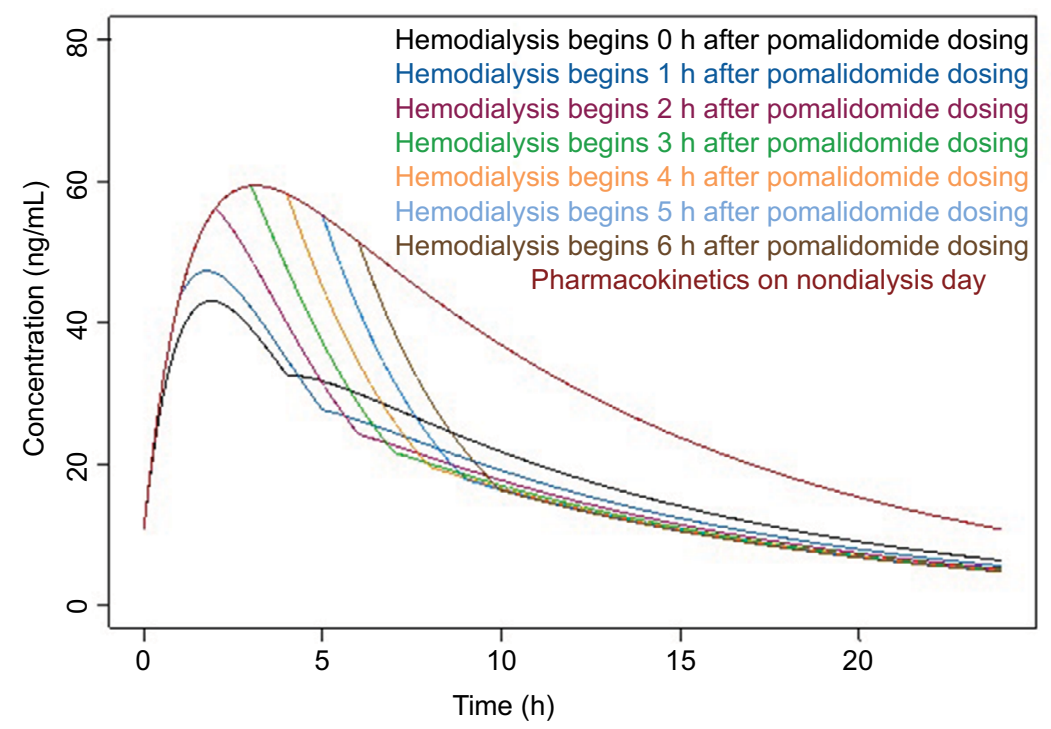

Figure 5 Simulated plasma concentration vs time profiles under different starting times of the hemodialysis procedure after pomalidomide administration.

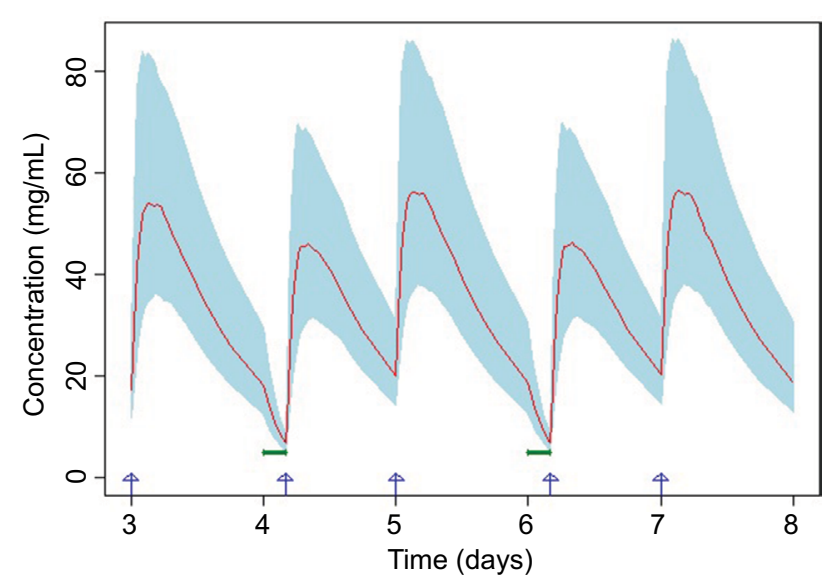

Figure 6 Simulated plasma concentration vs time profiles when the hemodialysis procedure is completed before pomalidomide administration.

Notes: Red line represents the mean of the simulated concentration vs time profile. Light blue shaded area represents the $90 \% \mathrm{Cl}$ of the simulated concentration vs time profile. Blue arrows represent pomalidomide dosing time. Bold green lines represent hemodialysis procedure (lasting for $4 \mathrm{~h}$ ).

comparable, and it was concluded that renal impairment did not significantly affect pomalidomide clearance over the $\mathrm{CrCl}$ range of 20 to $188 \mathrm{~mL} / \mathrm{min}$. This previous PPK analysis data set only included a limited number of patients with moderately or severely impaired renal function. Thus, there was a need for a thorough assessment of the influence of the impaired renal function on pomalidomide clearance, especially in patients with severely impaired renal function.

With an additional 63 patients with various degrees of renal impairment (eight patients with normal renal function, 15 with moderately impaired renal function, 30 with severely impaired renal function not requiring hemodialysis, and ten with severely impaired renal function requiring hemodialysis) from two clinical studies (CC-4047-MM-008 and CC-4047-MM-013), the effect of renal impairment on pomalidomide total body clearance and plasma exposure was critically re-examined in the current PPK analysis. Consistent with previous findings that renal function as measured by $\mathrm{CrCl}$ of 20 to $188 \mathrm{~mL} / \mathrm{min}$ did not remarkably affect pomalidomide clearance or plasma exposure, moderate or severe renal impairment did not affect pomalidomide clearance or plasma exposure. When renal function was regarded as a four-level categorical variable (group 1: normal renal function; group 2: moderately impaired renal function; group 3: severely impaired renal function not requiring hemodialysis; and group 4 : severely impaired renal function requiring hemodialysis), the analysis demonstrated that patients with moderately impaired renal function and patients with severely impaired renal function not requiring hemodialysis showed similar plasma clearance compared with patients with normal renal function $(\mathrm{CL} / \mathrm{F}$ ratios of $1.02[90 \%$ CI, 0.848-1.195] and 1.01 [90\% CI, 0.871-1.169], respectively) (Table 3). Patients with severely impaired renal function requiring hemodialysis had approximately $30 \%$ slower clearance compared with patients with normal renal function (CL/F ratio of 0.724 [ $90 \%$ CI, $0.606-0.844]$ ). None of the other tested covariates were significant enough to be included in the final model using the stepwise covariate model based on the predefined statistical criteria for inclusion of a covariate. Monte Carlo simulation results based on the final PPK model with renal function treated as a categorical variable demonstrated comparable pomalidomide exposures among the four groups of patients, further confirming that the difference in clearance was not small (Table 4). 
When renal function was treated as a continuous variable ( $\mathrm{CrCl}$ or eGFR), the analysis demonstrated that the intercept of the total pomalidomide clearance $(\mathrm{CL} / \mathrm{F})$ vs renal function marker ( 3.71 and $3.96 \mathrm{~L} / \mathrm{h}$ for $\mathrm{CrCl}$ and $\mathrm{eGFR}$, respectively) curve was approximately $70 \%$ of pomalidomide total clearance $(5.13 \mathrm{~L} / \mathrm{h})$, suggesting that non-renal clearance contributed roughly $70 \%$ of the total body clearance of pomalidomide, significantly more than renal clearance. In addition, the slope of the $\mathrm{CL} / \mathrm{F}$ vs renal function marker ( $\mathrm{CrCl}$ or eGFR) curve was shallow, consistent with the finding that pomalidomide was eliminated via non-renal clearance, and its total body clearance was not sensitive to changes in renal function (Table 5). Further, Monte Carlo simulation based on the final PPK model with renal function treated as a continuous variable showed consistent results with the Monte Carlo simulation based on the final PPK model with renal function treated as a categorical variable: the mean values of the plasma exposures (area under the plasma concentrationtime curve) were not remarkably different among the four groups of patients (group 1: patients with $\mathrm{CrCl}$ of $\geq 60 \mathrm{~mL} /$ $\min$; group 2: patients with $30<\mathrm{eGFR} \leq 45 \mathrm{~mL} / \mathrm{min} / 1.73 \mathrm{~m}^{2}$; group 3: patients with $\mathrm{CrCl}$ of $<30 \mathrm{~mL} / \mathrm{min}$ or eGFR of $<30$ $\mathrm{mL} / \mathrm{min} / 1.73 \mathrm{~m}^{2}$; group 4: patients with $\mathrm{CrCl}$ of $<15 \mathrm{~mL} /$ min or eGFR of $<15 \mathrm{~mL} / \mathrm{min} / 1.73 \mathrm{~m}^{2}$ ). Patients from group 4 showed mildly higher exposure $339.5 \%$ and $27 \%$ for $\mathrm{CrCl}$ and eGFR, respectively) compared with patients from group 1 (normal renal function group).

Analyses from hemodialysis data showed that the median values of the $\mathrm{CL}_{\mathrm{D}}$ were 2 -fold higher than pomalidomide total body clearance (12 and $5 \mathrm{~L} / \mathrm{h}$, respectively), indicating that the hemodialysis procedure removed pomalidomide from the blood. A simulation was conducted to optimize the start time of hemodialysis after pomalidomide administration. When the hemodialysis procedure began after pomalidomide administration, pomalidomide exposure on hemodialysis days was approximately $50 \%-70 \%$ of that on nondialysis days and was likely to result in insufficient pomalidomide exposures on hemodialysis days. However, when the hemodialysis procedure was completed before pomalidomide administration on hemodialysis days, pomalidomide exposure was approximately $83 \%-91 \%$ of that on nondialysis days and provided adequate pomalidomide exposures for patients with severely impaired renal function requiring hemodialysis.

In conclusion, this PPK analysis showed that there is no remarkable difference in pomalidomide exposures among different renal function groups, and there was a $35 \%$ higher exposure in patients with severe renal impairment requiring hemodialysis on nondialysis days. In addition, pomalidomide is cleared predominately via non-renal routes, accounting for approximately $70 \%$ of pomalidomide total body clearance. Thus, pomalidomide total body clearance is not sensitive to changes in renal function, as demonstrated by the relatively large intercept value (non-renal clearance) and shallow slope value in the relationship between pomalidomide $\mathrm{CL} / \mathrm{F}$ and renal function markers ( $\mathrm{CrCl}$ or eGFR). For patients with rrMM with end-stage renal disease requiring hemodialysis, the hemodialysis procedure removed pomalidomide from the blood; thus, pomalidomide should be administered after hemodialysis procedures.

\section{Acknowledgments}

This study was funded by Celgene Corporation. The authors would like to thank the patients and families as well as the investigators, nurses, and personnel who contributed to the CC-4047-MM-008 and CC-4047-MM-013 trials. The authors received editorial assistance from MediTech Media, Ltd, funded by Celgene Corporation.

\section{Disclosure}

Yan Li, Xiaomin Wang, Edward O'Mara, Elisabeth Kueenburg, Lars Sternas, Chloe Cavanaugh, Mohamed Zaki, Maria Palmisano, and Simon Zhou are employees of and hold equity ownership in Celgene Corporation; Meletios A Dimopoulos has received honoraria and consulting/advisory fees from Amgen, Celgene, Janssen, and Takeda; Pieter Sonneveld has received advisory/consultancy fees from Celgene, Amgen, Janssen, Karyopharm, and Skyline, and honoraria from Celgene, Amgen, Janssen, and Karyopharm; Katja C Weisel has received research funding from Celgene and Janssen and honoraria from Amgen, Bristol-Myers Squibb, Celgene, Janssen, Novartis, Onyx, and Takeda, and has held advisory board membership for Amgen, Bristol-Myers Squibb, Celgene, Janssen, Novartis, Onyx, and Takeda; Jeffrey Matous has received advisory/ consultancy fees from Celgene and served on their speaker's bureau; David S Siegel has served on speaker's bureaus for Celgene, Amgen, Takeda, Merck, and Novartis; Jatin J Shah has received research funding from Bristol-Myers Squibb, Celgene, Amgen, Novartis, and Array, consulting fees from Bristol-Myers Squibb, Celgene, Takeda, and Novartis, and served on speaker's bureaus for Bristol-Myers Squibb, Amgen, and Janssen. The authors report no other conflicts of interest.

\section{References}

1. Mitsiades N, Mitsiades CS, Poulaki V, et al. Apoptotic signaling induced by immunomodulatory thalidomide analogs in human multiple myeloma cells: therapeutic implications. Blood. 2002;99(12):4525-4530. 
2. Zhu D, Corral LG, Fleming YW, Stein B. Immunomodulatory drugs Revlimid (lenalidomide) and CC-4047 induce apoptosis of both hematological and solid tumor cells through NK cell activation. Cancer Immunol Immunother. 2008;57(12):1849-1859.

3. Hideshima T, Chauhan D, Shima Y, et al. Thalidomide and its analogs overcome drug resistance of human multiple myeloma cells to conventional therapy. Blood. 2000;96(9):2943-2950.

4. Verhelle D, Corral LG, Wong K, et al. Lenalidomide and CC-4047 inhibit the proliferation of malignant $\mathrm{B}$ cells while expanding normal CD34+ progenitor cells. Cancer Res. 2007;67(2):746-755.

5. Gupta D, Treon SP, Shima Y, et al. Adherence of multiple myeloma cells to bone marrow stromal cells upregulates vascular endothelial growth factor secretion: therapeutic applications. Leukemia. 2001;15(12):1950-1961.

6. Reddy N, Hernandez-Ilizaliturri FJ, Deeb G, et al. Immunomodulatory drugs stimulate natural killer-cell function, alter cytokine production by dendritic cells, and inhibit angiogenesis enhancing the anti-tumour activity of rituximab in vivo. Br J Haematol. 2008;140(1):36-45.

7. Lu L, Payvandi F, Wu L, et al. The anti-cancer drug lenalidomide inhibits angiogenesis and metastasis via multiple inhibitory effects on endothelial cell function in normoxic and hypoxic conditions. Microvasc Res. 2009;77(2):78-86.

8. Corral LG, Haslett PA, Muller GW, et al. Differential cytokine modulation and $T$ cell activation by two distinct classes of thalidomide analogues that are potent inhibitors of TNF-alpha. J Immunol. 1999;163(1):380-386.

9. Hayashi T, Hideshima T, Akiyama M, et al. Molecular mechanisms whereby immunomodulatory drugs activate natural killer cells: clinical application. Br J Haematol. 2005;128(2):192-203.

10. Teo SK, Chen Y, Muller GW, et al. Chiral inversion of the second generation IMiD CC-4047 (ACTIMID) in human plasma and phosphatebuffered saline. Chirality. 2003;15(4):348-351.

11. Daver N, Shastri A, Kadia T, et al. Phase II study of pomalidomide in combination with prednisone in patients with myelofibrosis and significant anemia. Leuk Res. 2014;38(9):1126-1129.

12. Richardson PG, Siegel DS, Vij R, et al. Pomalidomide alone or in combination with low-dose dexamethasone in relapsed and refractory multiple myeloma: a randomized phase 2 study. Blood. 2014;123(12):1826-1832.

13. San Miguel J, Weisel K, Moreau P, et al. Pomalidomide plus low-dose dexamethasone versus high-dose dexamethasone alone for patients with relapsed and refractory multiple myeloma (MM-003): a randomised, open-label, phase 3 trial. Lancet Oncol. 2013;14(11):1055-1066.
14. Pomalyst (pomalidomide). [Package insert]. Summit, NJ: Celgene Corporation; 2016.

15. Imnovid (pomalidomide). [Summary of product characteristics]. Uxbridge, UK: Celgene Europe; 2016.

16. Chen N, Wen L, Lau H, Surapaneni S, Kumar G. Pharmacokinetics, metabolism and excretion of [(14)C]-lenalidomide following oral administration in healthy male subjects. Cancer Chemother Pharmacol. 2012;69(3):789-797.

17. Yamasaki S, Kohno K, Kadowaki M, Takase K, Okamura S. Doseadjusted lenalidomide combined with low-dose dexamethasone rescues older patients with bortezomib-resistant multiple myeloma. Intern Med. 2015;54(14):1711-1715.

18. Chen N, Lau H, Kong L, Kumar G, Zeldis JB, Knight R, Laskin OL. Pharmacokinetics of lenalidomide in subjects with various degrees of renal impairment and in subjects on hemodialysis. J Clin Pharmacol. 2007;47(12):1466-1475.

19. Hoffmann M, Kasserra C, Reyes J, et al. Absorption, metabolism and excretion of [14C]pomalidomide in humans following oral administration. Cancer Chemother Pharmacol. 2013;71(2):489-501.

20. Li Y, Xu Y, Liu L, Wang X, Palmisano M, Zhou S. Population pharmacokinetics of pomalidomide. J Clin Pharmacol. 2015;55(5): 563-572.

21. Matous J, Siegel DS, Lonial S, et al. MM-008: A phase 1 trial evaluating pharmacokinetics and tolerability of pomalidomide + low-dose dexamethasone in patients with relapsed or refractory and refractory multiple myeloma and renal impairment. Blood. 2014;124(21):4730.

22. Sonneveld P, Weisel K, van de Donk N, et al. MM-013 phase 2 multicenter study of pomalidomide (POM) plus low-dose dexamethasone (LoDEX) in patients (pts) with relapsed/refractory multiple myeloma (RRMM) and renal impairment (RI). Haematologica. 2017;102(s2):P343.

23. Cockcroft DW, Gault MH. Prediction of creatinine clearance from serum creatinine. Nephron. 1976;16(1):31-41.

24. Levey AS, Bosch JP, Lewis JB, Greene T, Rogers N, Roth D. A more accurate method to estimate glomerular filtration rate from serum creatinine: a new prediction equation. Modification of diet in renal disease study group. Ann Intern Med. 1999;130(6):461-470.

25. Levey AS, Stevens LA, Schmid CH, et al. A new equation to estimate glomerular filtration rate. Ann Intern Med. 2009;150(9):604-612. 


\section{Supplementary material}

Table SI List of IECs/IRBs for CC-4047-MM-008 and CC-4047-MM-0I 3 studies

\begin{tabular}{|c|c|c|}
\hline Study & Site number & Name/address of IEC/IRB \\
\hline \multirow[t]{13}{*}{ CC-4047-MM-008 } & 001 & The University of Texas MD Anderson Cancer Center IRB, I400 Pressler, Unit 1437, Houston, TX \\
\hline & & $77030-4009$, USA \\
\hline & & The University of Texas MD Anderson Cancer Center Surveillance Committee FWA-363, I5I5 \\
\hline & & Holcombe Blvd, Unit 198, Houston, TX 77030-4009, USA \\
\hline & 002 & HCA-HealthONE Institutional Review Board, \\
\hline & & 4900 S. Monaco Street, 230 Denver, CO 80237, USA \\
\hline & 003 & Western Institutional Review Board, 1019 39th Ave SE, Suite 120, Puyallup, WA 98374, USA \\
\hline & & Western Institutional Review Board, 3535 Seventh Avenue, SW Olympia, WA 98502-50I0, USA \\
\hline & 004 & $\begin{array}{l}\text { Institutional Review Board of the Cleveland Clinic Foundation, } 9500 \text { Euclid Avenue OS-I, Cleveland, } \\
\mathrm{OH} 44195 \text {, USA }\end{array}$ \\
\hline & 006 & Emory University Institutional Review Board, 1599 Clifton Rd NE, 5th floor, Atlanta, GA 30322, USA \\
\hline & 100 & Local REB: \\
\hline & & Nova Scotia Health Authority Research Ethics Board, Centre for Clinical Research, 5790 University \\
\hline & & Avenue, Room II 8 Halifax, Nova Scotia, Canada B3H IV7 \\
\hline \multirow[t]{35}{*}{ CC-4047-MM-0I3 } & 101 & Comite de Protection des personnes OUEST III, CHU de la Miletrie, Pavilion Rene Le Blaye, Porte 9, 2 \\
\hline & & Rue de la Miletrie, BP 577, 86021 Poitiers Cedex, France \\
\hline & 103 & Comite de Protection des personnes OUEST III, CHU de la Miletrie, Pavilion Rene Le Blaye, Porte 9, 2 \\
\hline & & Rue de la Miletrie, BP 577, 8602 I Poitiers Cedex, France \\
\hline & 202 & Ethics Committee of the Medical Faculty of Eberhard Karis University and the University Hospital \\
\hline & & Tuebingen, Gartenstrasse 47, 72074 Tuebingen, Germany \\
\hline & 301 & CEC: \\
\hline & & Direction of Pharmaceutical Studies and Research, 284 Mesogeion str, Cholargos, II5 62 Athens, \\
\hline & & II528, Greece \\
\hline & & LEC: \\
\hline & & Ethics Committee of General Hospital of Athens "Alexandra", 80 Vas Sofias Ave and I Lourou str, \\
\hline & & Athens, II528, Greece \\
\hline & 401 & Comitato Etico Regionale delle Marche, Via Conca, n 7I, 60126 Ancona, Italy \\
\hline & & Formally known as: \\
\hline & & Comitato Etico AOU Ospedali Riuniti \\
\hline & 402 & Comitato Etico Provinciale di Reggio Emilia, Dipartmento Insfrastruttura Ricerca e Statistica, Azienda \\
\hline & & Ospedaliera Arcispedale Santa Maria Nuova/ IRCCS, Viale Umberto I, 50, 42 I23 Reggio Emilia, Italy \\
\hline & 403 & COMITATO ETICO BRIANZA, Via Pergolesi, 33 - 20090 Monza (MB), Italy \\
\hline & & Previous EC: \\
\hline & & Comitato Etico Interaziendale per le Province de Lecco Como e Sondrio, AO della Provincia di Lecco, \\
\hline & & Via dell'Eremo 9/I I, 23900 Lecco, Italy \\
\hline & 404 & Comitato Etico Milano Area 2, Segreteria Tecnico Scientifica ed Amministrativa, Fondazione IRCCS Ca' \\
\hline & & Granda Ospedale Maggiore Policlinico, Palazzo Uffici, Via F. Sforza n. 28, 20122 Milano, Italy \\
\hline & 501 & $\begin{array}{l}\text { Stichting Beoordeling Ethiek Biomedisch Onderzoek (Stichting BEBO), Stationsstraat 9, 940I KV Assen, } \\
\text { the Netherlands }\end{array}$ \\
\hline & 502 & $\begin{array}{l}\text { Stichting Beoordeling Ethiek Biomedisch Onderzoek (Stichting BEBO), Stationsstraat 9, 940I KV Assen, } \\
\text { the Netherlands }\end{array}$ \\
\hline & 601 & CEIC Área de Salud de Salamanca, Hospital Clínico Universitario - $2^{\mathrm{a}}$ planta Antiguo Edificio de \\
\hline & & Maternidad, Paseo de San Vicente, 58-182 \\
\hline & & 37007 Salamanca, Spain \\
\hline & 603 & $\begin{array}{l}\text { CEIC de l'Hospital Universitari Doctor Peset, Genoveva Pallarés Sancho-TelloC/ San Lázaro s/n. Edifici } \\
\text { de Riscos Laborals Ir Pis. } 46017 \text { València, Spain }\end{array}$ \\
\hline & 701 & North West Centre of Research Ethics Committees, Barlow House, 3rd Floor, 4 Minshull Street, \\
\hline & & Manchester, MI 3DZ, England \\
\hline & 702 & North West Centre of Research Ethics Committees, Barlow House, 3rd Floor, 4 Minshull Street, \\
\hline & & Manchester, MI 3DZ, England \\
\hline & 703 & North West Centre of Research Ethics Committees, Barlow House, 3rd Floor, 4 Minshull Street, \\
\hline & & Manchester, MI 3DZ, England \\
\hline
\end{tabular}


Table SI (Continued)

\begin{tabular}{lll}
\hline Study & Site number & Name/address of IEC/IRB \\
\hline 705 & $\begin{array}{l}\text { North West Centre of Research Ethics Committees, Barlow House, 3rd Floor, } 4 \text { Minshull Street, } \\
\text { Manchester, MI 3DZ, England }\end{array}$ \\
& Ethics Committee of the City of Vienna, Thomas-Klestil-Platz 8/2, I030 Vienna, Austria \\
801 & Ethics Committee of the Medical University of Vienna, Borschkegasse 8b / E06, I090 Vienna, Austria \\
802 & Kepler University Hospital, Ethics Committee of the Province of Upper Austria, Neuromed Campus, \\
& Wagner-Jauregg-Weg I5, A-4020 Linz, Austria \\
\hline
\end{tabular}

Abbreviations: CEC, central ethics committee; CEIC, Comité Ético de Investigación Clínica [clinical research ethics committee]; EC, ethics committee; FWA, Federalwide Assurance; IEC, institutional ethics committee; IRB, institutional review board; LEC, local ethics committee; REB, research ethics board. peer-reviewed, open access journal publishing original research, reports,

Visit http://www.dovepress.com/testimonials.php to read real quotes reviews and commentaries on all areas of drug experience in humans.

The manuscript management system is completely online and includes a very quick and fair peer-review system, which is all easy to use. 